Paediatric rheumatology

\section{AB1078 APPLICABILITY OF THE CASPAR CRITERIA OF PSORIASIC ARTHRITIS IN A COHORT OF CHILDREN PATIENTS FOLLOWING IN A PAEDIATRIC RHEUMATOLOGY UNIT OF A TERTIARY HOSPITAL}

A. Alía Jiménez, A. Lucica Boteanu, B.A. Blanco Cáceres, M. Blázquez Cañamero. Rheumatology, Hospital Ramón y Cajal, Madrid, Madrid, Spain

Background: The ILAR consensus establishes classification criteria, dividing the JIA into 7 subcategories, with juvenile psoriatic arthritis (APsJ) being one of them In the adult population, the CASPAR classification criteria are usually used to classify a patient with psoriatic arthritis. However, the two classifications have some differences that sometimes produce confusion

Objectives: To assess the applicability of the CASPAR classification criteria in a series of patients previously diagnosed in paediatric age of JPAs or undifferentiated arthritis by exclusion criteria to be male $>6$ years old and HLA B27 positive, comparing these with the ILAR classification criteria, through the study of clinical features

Methods: Retrospective cross-sectional observational study. Clinical, epidemiological, sociodemographic and analytical variables were collected from 30 patients previously diagnosed with JPAs ( $<16$ years) or undifferentiated arthritis by exclusion criteria age $>6$ years in HLA B27-carrying male. It was assessed whether the patients met the ILAR classification criteria as well as the CASPAR classification criteria, which, unlike the previous ones, did not exclude HLA B27 positive patients, considered the family history of the 2 nd degree and added a test radiographic

Results: The mean age at diagnosis was $11.23 \pm 4.6$ years; 15 of them being women and 15 men. 15 (15/30) patients presented cutaneous psoriasis at some point during the follow-up, in $5 / 15$ patients psoriasis began before arthritis while $7 /$ 15 patients were previously diagnosed with arthritis than cutaneous psoriasis; in $3 / 15$ patients the diagnosis was simultaneous during the medical visit. $9(9 / 30)$ patients presented a family history of 1 st degree cutaneous psoriasis and $7 / 30$ of them had a family history of 2 nd grade psoriasis. Of the total number of patients, 10 of them would not meet the ILAR classification criteria, 8 because they presented as exclusion criteria being male, HLA-B27 positive and $>6$ years of age, among which, $7 / 8$ would fulfil CASPAR criteria, and 2 other patients who were not classified according to ILAR criteria, did meet the CASPAR classification criteria, given the presence in these criteria of negative RF, family history of the 2nd degree and typical radiological alterations, which are not present in the ILAR criteria. $1(1 / 30)$ patient did not meet CASPAR criteria, and belonged to the group of patients excluded from the ILAR criteria for being male $>6$ years HLA-B27 +

If we did not take into account the negative FR of the CASPAR criteria, 14 patients would not meet these criteria and if we eliminated the 2 nd grade AF, 5 patients would not be classified (among them 2 who meet CASPAR and do not ILAR)

Conclusions: In our series of patients despite the fact that the presence of current skin psoriasis contributes 2 points in the CASPAR criteria, only 1 patient would not meet the CASPAR criteria, since the majority of patients present other clinical or analytical manifestations, such as the presence of negative rheumatoid factor or 2nd degree family history. Patients who do not meet criteria for PsA by exclusion criteria, practically all of them would be diagnosed with psoriasic arthritis by CASPAR criteria.

Disclosure of Interest: None declared

DOI: 10.1136/annrheumdis-2018-eular.7372

\section{AB1079 TRANSITION CARE OF PATIENTS WITH CHILDHOOD ONSET CHRONIC RHEUMATIC DISEASE IN A TERTIARY MEDICAL CENTRE IN TURKEY}

A. Adrovic ${ }^{1}$, E. Pehlivan ${ }^{1}$, O. Kul1 ${ }^{1}$, S. Sahin ${ }^{1}$, K. Barut ${ }^{1}$, S. Ugurlu² , H. Ozdogan ${ }^{2}$ O. Kasapcopur $1 .{ }^{1}$ Pediatric Rheumatology, ${ }^{2}$ Rheumatology, Istanbul University, Cerrahpasa Medical School, Istanbul, Turkey

Background: Transitional care is a purposeful, planed movement of adolescence and young adults with chronic condition from childhood- to adult-oriented health care systems. Well-organised, systematic transitional health care is of high importance for providing the continuous medical treatment and for reaching optimal outcomes. Up to date, there is no unique, consensus-based model for patients' transition from childhood- to adult oriented health care centres in Turkey. Objectives: We aimed to assess the transitional care among patients with juvenile onset rheumatologic disease in referral tertiary centre in Turkey.

Methods: The transitional policlinic is held once per month at the department for paediatric rheumatology, consisting of 2 adult and 4 paediatric rheumatologist. A total of 147 patients have undergone the transitional care in the time period from May 2014 to December 2017. Patient s were called by telephone, by two different investigators (EP, OK). Thirty-three (22\%) patients were not reached and 17 $(11 \%)$ of theme were excluded from the study due to short post-transitional period
(<6 months). Consequently, 97 (66\%) patients have been reached and included in the study. Data on demographic, clinical and socio-economic features and experience with transitional practice were collected by using a structured questionnaire, which was fulfilled during the phone conversation between investigator and patient.

Results: A total of 147 patients $(79(54 \%)$ females) underwent transition process and 97 of them were included in the study. There was no statistically significant difference between different patients groups regarding the age of transition. The education levels of patients were as following: university $60(61.9 \%)$, high-school $21(21.6 \%)$, middle-school $13(13.4 \%)$, primary school $3(3.1 \%)$. Majority of patients was single at the time of study $(79(81.4 \%)$ patients) while only 18 $(18.6 \%)$ patients were married; half of them being child owner. At the time of study, $44(30 \%)$ patients were employed and mean age at employment was $19.06 \pm 3.1$ years. Most of patients had health insurance at the time of study (94 (96.9\%)) Seventy-one $(73.2 \%)$ patients continued their regular follow up at adult department while $26(25.8 \%)$ patients discontinued medical treatment. The most com mon reasons for cessation of follow up were the work/school absence (20 $(76.9 \%))$, followed by patients' personal reasons $(2(7.6 \%))$ and dissatisfaction with adult clinic services $(4(15.5 \%))$. Most of patients reported satisfaction with transition process: 96 (99\%).

Abstract AB1079 - Table 1

\begin{tabular}{lc}
\hline & \\
\hline Female, $\mathrm{n}(\%)$ & $\mathrm{n}=97$ \\
Age at transition, mean \pm SD & $58(59.7 \%)$ \\
Age at study mean \pm SD & $21.4 \pm 1.4$ years \\
JIA & $22.8 \pm 1.8$ years \\
FMF & $26(26.8 \%)$ \\
Connective & $60(61.8 \%)$ \\
Tissue & $7(7 \%)$ \\
Disease & \\
Vasculitis & \\
\hline
\end{tabular}

Conclusions: This is the first Turkish model of transitional care among patients with chronic rheumatic disease. Well-organised transitional health care is of crucial importance for the continuous and optimal health care of adolescents and young adults with chronic rheumatic disease. Further studies with higher number of patients would reveal the relevance of described transitional care model.

\section{REFERENCE:}

[1] - Foster HE, Minden K, Clemente D, et al. EULAR/PReS standards and recommendations for the transitional care of young people with juvenileonset rheumatic diseases. Ann Rheum Dis. 2017;76:639-646.

Disclosure of Interest: None declared

DOI: 10.1136/annrheumdis-2018-eular.3437

\section{AB1080 NEUROLOGICAL EVALUATION OF CHILDHOOD-ONSET CRYOPYRIN-ASSCOCIATED PERIODIC SYNDROMES-A PRELIMINARY REPORT}

H. Kilic ${ }^{1}$, S. Sahin ${ }^{2}$, C. Duman ${ }^{1}$, A. Adrovic ${ }^{2}$, K. Barut ${ }^{2}$, O. Kasapcopur², S. Saltik ${ }^{1}$ ${ }^{1}$ Pediatric Neurology, ${ }^{2}$ Pediatric Rheumatology, Istanbul University, Cerrahpasa Medical School, Istanbul, Turkey

Background: The cryopyrin-associated periodic syndrome (CAPS) is a treatable autoinflammatory disease that encompasses familial cold autoinflammatory syndrome(FCAS), Muckle-Wells syndrome(MWS), and chronic infantile, neurologic, cutaneous, and articular syndrome (CINCA), which are quite different in severity. Early diagnosis of CAPS and prompt initiation of IL-1 blockers have significant effect on the neurologic prognosis of CAPS. Although neurologic complications of CINCA are well-known, there are scarce date regarding neurologic features of milder phenotypes.

Objectives: We aimed to review the neurologic features in detail and summarise the other CAPS-related manifestations in 9 children.

Methods: All children with CAPS that have been followed-up from paediatric rheumatology outpatient clinic, were enrolled to the study. In addition to the neurologic examination, magnetic resonance imaging (MRI) of brain, electroencephalography, eye examination, hearing test and neuropsychiatric tests were done. Demographic, clinical features, genetic analysis and laboratory tests were noted from patient records and hospital database.

Results: The median age of the subjects was 6 years (range 2-14 years), with a female-to-male ratio $4 / 5$. Most frequently noted neurologic clinical manifestations during the disease course were papilledema (3/9) and epilepsy (3/9), followed by neurodevelopmental delay (2/9), aseptic meningitis (2/9), upper motor neuron 